\title{
A Multi-Microscopy Approach to Discover the Feeding Site and Host Tissue Consumed by Varroa destructor on Host Honey Bees
}

Samuel Ramsey ${ }^{1}$, Connor J. Gulbronson ${ }^{2}$, Joseph Mowery ${ }^{3}$, Ron Ochoa ${ }^{4}$, Dennis vanEngelsdorp ${ }^{1}$, and Gary Bauchan ${ }^{3}$,

1. Department of Entomology, University of Maryland, College Park, MD 20742 USA

2. Floral and Nursery Plant Research Unit, United States Department of Agriculture, Agricultural Research Service (USDA-ARS), U.S. National Arboretum, Beltsville, MD 20705, USA and Oak Ridge Institute for Science and Education (ORISE) Postdoctoral Fellow.

3. Electron and Confocal Microscopy Unit, USDA-ARS, Beltsville, MD 20705, USA

4. Systematic Entomology Laboratory, USDA-ARS, Beltsville, MD 20705, USA

Honey bees have experienced a global trend of colony losses due in large part to the parasitic mite Varroa destructor. For the past 5 decades, the conclusion that Varroa feed exclusively on the hemolymph of host bees has been the accepted view among researchers and beekeepers despite a lack of experimental evidence [1]. We have conducted studies to determine if Varroa feed on hemolymph or the proposed alternative tissue, fat body. Four different microscopy systems including Low Temperature Scanning Electron Microscopy (LT-SEM), Transmission Electron Microscopy (TEM), Confocal Laser Scanning Microscopy (CLSM) and Fluorescence Microscopy were used to determine where these mites feed and what tissue they ingest.

Low Temperature Scanning Electron Microscopy (LT-SEM) was performed by freezing mite parasitized bees using a Quorum 2000 PT Cryo-Preparation system attached to a Hitachi 4700 FE SEM [2]. Bees with these attached mites were affixed to copper plates with cryo-glue and imaged. Following imaging of the location of the mite (Figure 1A), the mite was removed from the bee while still frozen and the bee returned to the SEM. An imprint of the mite remained on the bee, revealing a feeding hole in the intervening membrane between the overlapping abdominal plates (Figure 1B). This provided the first direct evidence that Varroa mites feed on adult bees. Additionally, cryo-freeze fractures were performed in order to obtain a cross-section of the feeding site of the mite on the bee. Freeze fractures coupled with separate observations via light microscopy revealed that the tissue beneath this membrane is the fat body (Figure 1C).

Transmission electron microscopy (TEM) studies were conducted using a Hitachi 7700 TEM [3]. Mites, which were actively feeding on bees, were affixed in place with cyanoacrylate glue prior to fixation to insure the mites remained attached to the bee during the fixation and embedding process (Figure 1D). Thick sections and ultra-thin sections of the feeding site revealed a hole with irregular edges where the mouth parts of the mite had penetrated the bee's intersegmental membrane. Degraded fat body cells were found directly beneath the intersegmental membrane, likely due to the extra-oral digestion of the mite at the feeding site. Two species of morphologically distinct bacteria were also imaged in the feeding wound suggesting mechanical vectoring by the mite (Figure 1E). Varroa destructor is also known to vector several species of viruses with have been shown to affect bee health.

Confocal Laser Scanning Microscopy (CLSM) studies were conducted using a Zeiss LSM710 CLSM as well as Fluorescence Microscopy using a Zeiss AxioZoom.V16 microscope. Bees were fed a fluorescent lipophilic biostain, Nile Red (red), which persisted in the fat body tissue and a fluorescent hydrophilic 
biostain, Uranine (yellow), which persisted in the hemolymph. Mites that fed on bees with a diet of only the hydrophilic Uranine, showed fluorescence levels similar to the control (given no biostain), however, fluorescence associated with honey bee fat body tissue was consistently detected inside the gut of the mites (Figure 1F).

$V$. destructor feeds on fat body tissue rather than blood, a fact with broad implications for the fields of honey bee virology, apiculture, and pesticide development. Collectively, the findings obtained using these varied microscopy technologies fundamentally alter our understanding of this mite underscoring a need to reanalyze even the basics of how we understand this economically significant host/parasite relationship.

[1] P. Rosenkranz, P. Aumeier, \& B. Ziegelmann J. Inv. Path. 103 (2010) p. 96-119.

[2] S. J. Bolton et al. J. Nat. History 48 (2014) p. 1359-1373.

[3] J. Trupkiewicz et al. Veterinary Parasitology 216 (2016) p. 52-58.

Mention of trade names or commercial products in this publication is solely for the purpose of providing specific information and does not imply recommendation or endorsement by the U.S. Department of Agriculture. USDA is an equal opportunity provider and employer.

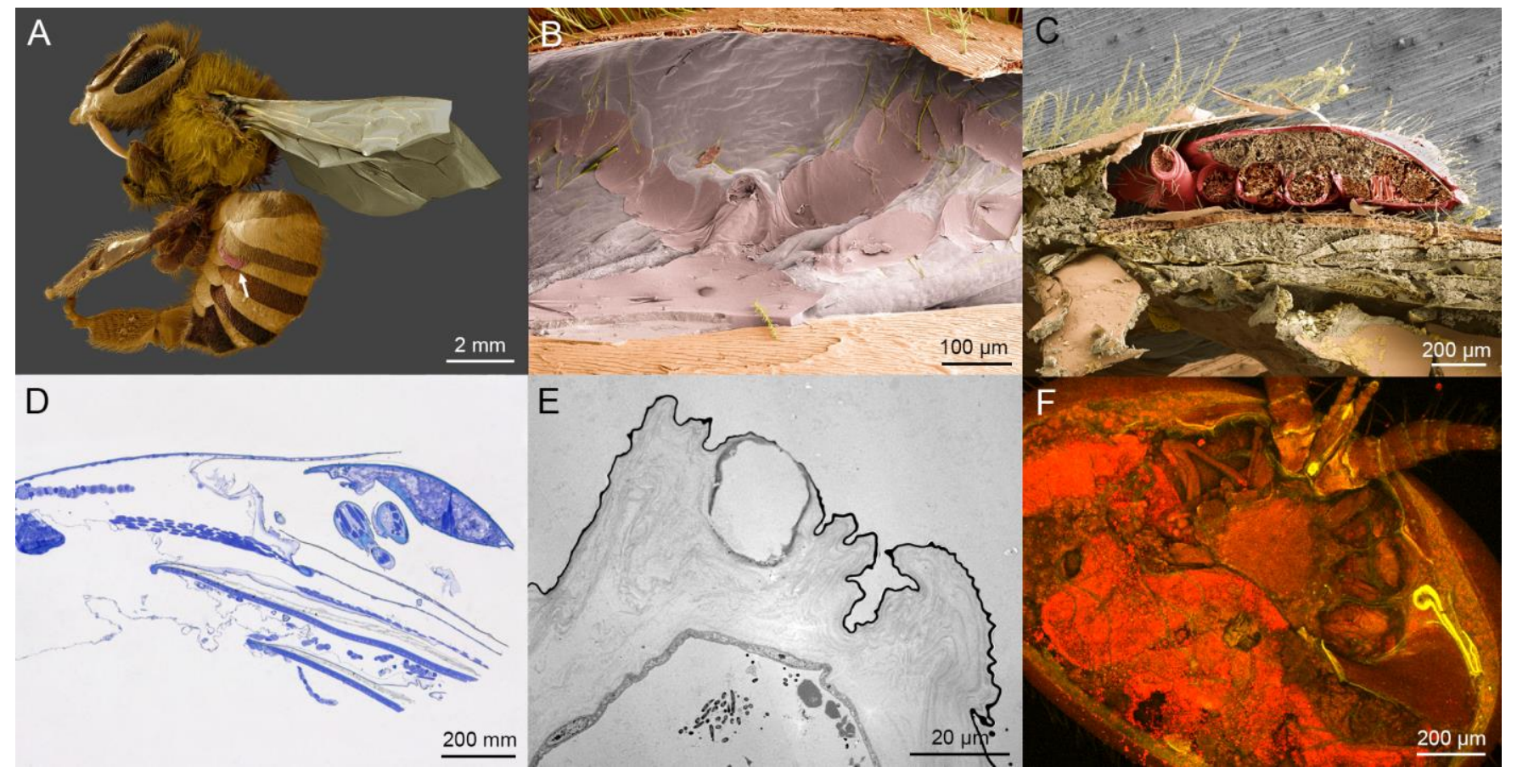

Figure 1. Microscopy Images of Honey Bees and Varroa. A. Colorized low temperature scanning electron microscopy image of the location of the primary feeding site of Varroa (see arrow); B. Colorized LTSEM image of the imprint of a Varroa mite on the intersegmental membrane where the mite was feeding; C. Colorized LT-SEM image of a cross-section of a Varroa mite on a bee (mite is colorized red); D. Bright field image of a thick section of a mite feeding on a bee (mite in upper right corner); E. Transmission electron microscopy image of the feeding site (hole) and small bacteria beneath the membrane, F. Confocal Laser Scanning Microscopy image of the gut of a mite brightly stained after ingesting biostained fat body tissue from a host bee with differentially biostained hemolymph and fat body tissue. 